\title{
Neuromyelitis optica spectrum disorder and Sjögren syndrome are overlapping disorders and participate in the same autoimmunity context?
}

\author{
Síndrome de Sjögren e afecções do espectro da neuromielite óptica fazem parte do \\ mesmo contexto autoimune?
}

Maria Lucia Vellutini Pimentel ${ }^{1,2}$

${ }^{1}$ Departamento Científico de Neuroimunologia, Academia Brasileira de Neurologia, Sao Paulo SP, Brazil;

${ }^{2}$ Departamento de Neurologia, Santa Casa da Misericórdia, Rio de Janeiro RJ, Brazil.

Correspondence:

Maria Lucia Vellutini Pimentel; Rua Garcia D'Avila, 64 - sala 205; 22421-010 Rio de Janeiro RJ, Brasil; E-mail: mlvpimentel@gmail.com Conflict of interest:

There is no conflict of interest to declare.

Received 09 June 2014 Accepted 27 June 2014

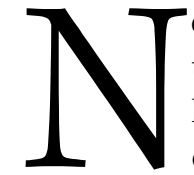
euromyelitis optica (NMO), an idiopathic inflammatory demyelinating disease of the central nervous system that mainly affects the optic nerves and spinal cord, has long been thought to be a variant of multiple sclerosis (MS), although its clinical evolution and hystopathological findings had many particularities. Also, recurrent demyelinating diseases affecting the optical nerve and spinal cord, differently from MS, had signs of hypothalamic and hypophyseal dysfunction and a common association with other systemic autoimmune diseases ${ }^{1,2,3,4,5,6,7}$.

Only in 2004, NMO had been recognized as a unique demyelinating disease ${ }^{8}$ when Lennon et al. ${ }^{9}$ identified a serum autoantibody marker in patients with NMO, named NMO-IgG (AQP4-IgG), that bound selectively to the aquaporin 4 (AQP4) water channels which is concentrated in the astrocytic foot processes at the blood-brain barrier. It would be not merely a marker but a causative agent ${ }^{10}$.

In 2006, after the description of the NMO-IgG, Wingerchuk DM et al. ${ }^{11}$ revised the diagnostic criteria for NMO that comprised symptoms related to optical neuritis and myelitis which should be longitudinally extensive, associated with a brain MRI, now acceptable to have demyelinating lesions although without the same distribution as the ones seen in MS, and the presence of NMO-IgG.

On the other hand, NMO-IgG was also detectable in the serum of patients with disorders related to NMO, as recurrent myelitis with longitudinally extensive spinal cord lesions, recurrent isolated optic neuritis, myelitis or optic neuritis in the context of certain organspecific diseases as Sjögren syndrome (SS) or systemic lupus erythematosus (SLE), sarcoidosis, Behçet disease, and non-organ-specific autoimmune diseases (antinuclear antibody, extractable nuclear antigen) which were named the spectrum of neuromyelitis optica (NMOSD), and also in Asian optic-spinal $\mathrm{MS}^{9,10,11,12}$. The percentage of patients fulfilling the diagnostic of NMOSD is low (1-2\%) in white people from Europe, North America, or Australia and high (20-48\%) in people from West Indies and Asia ${ }^{12,13,14}$.

Over the last two decades, cases of isolated recurrent myelitis, characteristically a longitudinal transverse myelitis, the combination of myelitis and optic neuritis suggestive of NMO were associated to SLE or SS ${ }^{11,12,13,14,15,16}$. Patients with NMO have often autoantibodies or autoimmune diseases and SS is the most frequent autoimmune disorder seen in these patients. The existence of SS in patients with NMOSD was estimated to be between 2 and $30 \%$ and myelopathy is described in 20 to $34 \%$ of all patients with SS, being occasionally the initial manifestation; 7.7 to $12 \%$ of patients with NMO have anti-SS-A or anti-SS-B antibodies. Investigating for AQP4 antibodies in patients diagnosed with SS who developed longitudinal transverse myelitis is an important data for prognosis and treatment ${ }^{16,17,18,19}$.

Pittock SJ et al. ${ }^{15}$ evaluated the association of NMO-IgG and non-organ-specific auto antibodies in patients with SS and NMOSD and, considering the specificity of NMO-IgG for 
NMO, proponed three hypothesis: 1) NMOSDs and SS/SLE are non overlapping conditions complicated by optic neuritis and myelitis, 2) these conditions are independent and coexist in the same patient and 3) the serological findings are non specific and NMO-IgG or non-organspecific antibodies may be seen in either condition. The authors demonstrated that NMO and SS are overlapping disorders that coexist in the same patient although, for better understanding of the relationship of these diseases, they would need the immunopathological analysis of optical nerve and spinal cord lesions which, until then, there were not any. But later, Kim et al. ${ }^{17}$ found that spinal cord involvement in SS were likely to be a manifestation of NMO rather than a direct compromise of the central nervous system in SS patients because of the vasculitis induced by the connective tissue disorders which should create the inflammatory environment for the inflammatory cascade mediated by anti-AQP4.

In this number of Arquivos de Neuro-Psiquiatria, Carvalho DC et al. revised the literature in search of the relationship among AQP4-IgG serum status, NMOSD and $\mathrm{SS}^{20}$. To illustrate their understanding of the coexistence of SS and NMOSD as a predisposition of patients with NMO to multiple autoimmune diseases, they described two cases of primary SS and definite NMO with different AQP4-IgG serum status and other non-organ-specific auto antibodies. Both cases were women in their forties; in Case 1 the sicca syndrome developed long before NMO and in Case 2 recurring optic neuritis occurred few years before the manifestations of SS. The serum AQP4-IgG was negative in Case 1 and positive in Case 2. They explained that different methods of investigation could not be sensitive enough to detect the AQP4-IgG or it could remain negative due to treatment or remission of the disease, which is in accordance with the literature ${ }^{12}$. An improvement in assay sensitivity would increase the number of serum AQP4-IgG positive to fulfill the diagnostic of NMO or NMOSD. They also report evidence of the clinical and epidemiological differences between AQP4-IgG positive and AQP4-IgG negative neuromyelitis optica and emphasize the importance to check for this antibody for treatment decision and prognostic information.

Carvalho et al. suggested in this article that SS and NMOSD should coexist in the same milieu of autoimmunity, independently from the AQP4 autoimmunity and also that anti-MOG ${ }^{21}$ could play a role in NMOSD patients who are AQP4-IgG negative. They have properly pointed out the difficulties that still are regarding the understanding of NMO and NMOSD in the context of other autoimmune diseases and have shown how careful we should be in the investigation and treatment decision regarding these patients.

Finally they concluded that further research is necessary to elucidate the role of AQP4-IgG and anti MOG in NMOSD patients with SS disease.

\section{References}

1. Fazekas F, Offenbacher H, Schmidt R, et al. MRI of neuromyelitis optica: evidence for a distinct entity. J Neurol Neurosurg Psychiatry 1994;57:1140-1142.

2. Kira J, Harada M, Yamagushi Y, et al. Hyperprolactinemia in multiple sclerosis. J Neurol Sci 1991;102:61-66.

3. Vernant JC, Cabre P, Smadja D, et al. Recurrent optic neuromyelitis with endocrinopathies: a new syndrome. Neurology 1997;48:58-64.

4. Wingerchuk DM, Hogancamp WF, OáBrien PC, Weinshenker BG. The clinical course of Neuromyelitis optica (Devicás syndrome). Neurology 1999;53:1107-1114.

5. Scolding N. Devicás disease and autoantibodies: reflection and reaction. The Lancet Neurology 2005;4:136-137.

6. De Seze J, Lebrun C, Stojkovic T, et al. Is Devicás neuromyelitis optica a separate disease? A comparative study with multiple sclerosis. Multiple Sclerosis 2003;9:521-525.

7. Popescu BFGh, Lennon VA, Parisi JE, et al. Neuromyelitis optica unique area postrema lesions. Nausea, vomiting, and pathogenic implications. Neurology 2011;76:1229-1237.

8. Matiello M, Jacob A, Wingerchuk DM and Weinshenker BG. Neuromyelitis optica. Curr Opin Neurol 2007;20:255-260.

9. Lennon VA, Wingerchuck DM, Kryzer TJ, et al. A serum autoantibody marker of neuromyelitis optica: distinction from multiple sclerosis. Lancet 2004;364:2106-2112.

10. Cross SA. Rethinking neuromyelitis optica (Devic disease). J NeuroOphtalmol 2007;27:57-60.
11. Wingerchuk DM, Lennon VA, Pittock SJ, Lucchinetti CF, Weinshenker BG. Revised diagnostic criteria for neuromyelitis optica. Neurology 2006;66:1485-1489.

12. Wingerchuk DM, Lennon VA, Lucchinetti CF, et al. The spectrum of neuromyelitis optica. Lancet Neurology 2007;6:805-815.

13. Wingerchuk DM, Weinshenker BG. The emerging relationship between neuromyelitis optica and systemic autoimmune disease. MSJ 2012;18:5-10.

14. Papadopoulus MC, Verkman AS. Aquaporin 4 and neuromyelitis optica. Lancet Neurology 2012;11:535-544.

15. Pittock SJ, Lennon VA, De Seze J, et al. Neuromyelitis optica and nonorgan-specific autoimmunity. Arch Neurol 2008;65:78-83.

16. Pittock SJ, Lennon VA, Wingerchuk DM, et al. The prevalence of nonorgan-specific autoantibodies and NMO-IgG in Neuromyelitis optica and related disorders. Neurology 2006;66(Suppl 2):S307.

17. Kim SM, Waters P, Vincent A, et al. Sjögren's syndrome myelopathy: spinal cord involvement in Sjögren's syndrome might be a manifestation of neuromyelitis optica. Multiple Sclerosis 2009;15:1062-1068.

18. Jarius S, Jacobi Christian, De Seze J, et al. Frequency and syndrome soecificity of antibodies to aquaporin- 4 in neurological patients with rheumatic disorders. MSJ 2011;17:1067-1073.

19. Kahlenberg JM. Neuromyelitis optica spectrum disorders as an initial presentation of primary Sjögren's syndrome. Semin Arthritis Rheum 2011;40:343-348. 
20. Carvalho DC, Tironi TS, Freitas DS, Kleinpaul R, Talim NC, LanaPeixoto MA. Sjögren syndrome and neuromyelitis optica spectrum disorders co-exist in a common autoimmune milieu. Arq Neuropsiquiatr 2014;72:619-624.
21. Sato DK, Callegaro D, Lanna-Peixoto MA, et al. Distinction between MOG antibody positive and AQP4 positive NMO spectrum disorders. Neurology 2014;82:474-481. 